\title{
OPEN SOURCE WEB TOOL FOR TRACKING IN A LOW COST MOBILE MAPPING SYSTEM
}

\author{
F. Fissore ${ }^{\mathrm{a},},{ }^{\text {F. Pirotti }}{ }^{\mathrm{a}}$, A. Vettore ${ }^{\mathrm{a}}$ \\ ${ }^{\text {a }}$ Interdepartmental Research Center of Geomatics (CIRGEO), University of Padova, \\ Viale dell'Università 16, Legnaro (PD) 35020, Italy - \\ (francesca.fissore, francesco.pirotti, antonio.vettore)@unipd.it
}

KEY WORDS: Web-tool, Mobile mapping, Low-cost, Open source, Inertial navigation system

\begin{abstract}
:
During the last decade several Mobile Mapping Systems (MMSs), i.e. systems able to acquire efficiently three dimensional data using moving sensors (Guarnieri et al., 2008, Schwarz and El-Sheimy, 2004), have been developed. Research and commercial products have been implemented on terrestrial, aerial and marine platforms, and even on human-carried equipment, e.g. backpack (Lo et al., 2015, Nex and Remondino, 2014, Ellum and El-Sheimy, 2002, Leica Pegasus backpack, 2016, Masiero et al., 2017, Fissore et al., 2018). Such systems are composed of an integrated array of time-synchronised navigation sensors and imaging sensors mounted on a mobile platform (Puente et al., 2013, Tao and Li, 2007). Usually the MMS implies integration of different types of sensors, such as GNSS, IMU, video camera and/or laser scanners that allow accurate and quick mapping (Li, 1997, Petrie, 2010, Tao, 2000). The typical requirement of high-accuracy 3D georeferenced reconstruction often makes such systems quite expensive. Indeed, at time of writing most of the terrestrial MMSs on the market have a cost usually greater than 50000, which might be expensive for certain applications (Ellum and El-Sheimy, 2002, Piras et al., 2008). In order to allow best performance sensors have to be properly calibrated (Dong et al., 2007, Ellum and El-Sheimy, 2002).

Sensors in MMSs are usually integrated and managed through a dedicated software, which is developed ad hoc for the devices mounted on the mobile platform and hence tailored for the specific used sensors. Despite the fact that commercial solutions are complete, very specific and particularly related to the typology of survey, their price is a factor that restricts the number of users and the possible interested sectors.

This paper describes a (relatively low cost) terrestrial Mobile Mapping System developed at the University of Padua (TESAF, Department of Land Environment Agriculture and Forestry) by the research team in CIRGEO, in order to test an alternative solution to other more expensive MMSs. The first objective of this paper is to report on the development of a prototype of MMS for the collection of geospatial data based on the assembly of low cost sensors managed through a web interface developed using open source libraries. The main goal is to provide a system accessible by any type of user, and flexible to any type of upgrade or introduction of new models of sensors or versions thereof. After a presentation of the hardware components used in our system, a more detailed description of the software developed for the management of the MMS will be provided, which is the part of the innovation of the project. According to the worldwide request for having big data available through the web from everywhere in the world (Pirotti et al., 2011), the proposed solution allows to retrieve data from a web interface Figure 4. Actually, this is part of a project for the development of a new web infrastructure in the University of Padua (but it will be available for external users as well), in order to ease collaboration between researchers from different areas.
\end{abstract}

Finally, strengths, weaknesses and future developments of the low cost MMS are discussed.

\section{INTRODUCTION}

Mobile Mapping System (MMS) is a technology able to acquire in an efficient way three dimensional data able to describe with accuracy the surrounding environment (Guarnieri et al., 2008, Schwarz and El-Sheimy, 2004). Such systems are composed of an integrated array of time-synchronised navigation sensors and imaging sensors mounted on a mobile platform (Puente et al., 2013, Tao and Li, 2007). Usually this thecnology integrates different types of sensors (e.g. GNSS, IMU, video camera, laser scanners etc.) that allow accurate and quick mapping ( $\mathrm{Li}, 1997$, Petrie, 2010, Tao, 2000).

Nowadays most of the MMSs available on the market have a cost usually greater than 50000 (Ellum and El-Sheimy, 2002, Piras et al., 2008) and typically they are composed by an integrated system of sensors managed through dedicated software which are developed for the devices mounted on the mobile platform and specific for the sensors used. Some examples of commercial MMSs are the following: Mitsubishi MMS-X640 Mobile Map-

${ }^{*}$ Corresponding author. ping System, RIEGL VMX-250 Mobile Laser Scanning System, StreetMapper 360, LYNX Mobile Mapper, IP-S2 Mobile Mapping System, Teccon Mobile Mapper and MDL Dynascan Mobile Mapping System. Despite the fact that commercial solutions have high-quality performances their price is a factor that restricts the number of users and the possible interested sectors.

This paper will describe an alternative model of Mobile Mapping System, developed at the University of Padua (TESAF, Department of Land Environment Agriculture and Forestry) by the research team in CIRGEO. The objective of this paper is to report on the development of a prototype of MMS for the collection of geospatial data based on the assembly of low cost sensors managed through a web interface developed using open source libraries. The main goal is to build a system accessible by any type of user, and flexible to any type of upgrade or introduction of new models of sensors or versions thereof. The following pages will describe the MMS system and its components, will show the libraries and components of the web interface, and discuss strengths and weaknesses. After a presentation of the hardware components used in our system, a more detailed description of the software developed for the management of the MMS follows, 
which is the part of the true innovation of the project. In the final part possible future developments of the low cost MMS will be discussed.

\section{CONFIGURATION OF LOW COST MMS}

The idea to the base of this research is the development of a prototype of MMS composed by the assembling of low cost sensors managed through a open source software which has the appearance of a web page.

All the sensors are installed on a rigid platform created to be small in size, easy to transport and adjustable to different models of vehicles (see Figure 1).

The choice of using low cost sensors is done to reduce the price of MMS making it accessible to a wider range of users, while the management of the system through web interface will facilitate its usage during the data acquisition, storage and synchronization. Furthermore, the use of open source software for the web interface dedicated to processing enables easier integration of new sensors in our MMS at later stages.

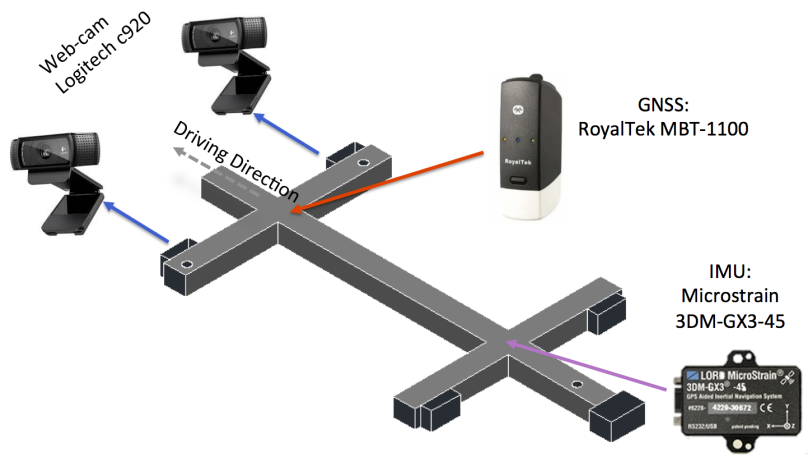

Figure 1. Layout of rigid platform.

\subsection{Software}

The most important goal to reach in the development of a Mobile Mapping System is to collect and synchronize the data coming from navigation and imaging sensors. For that purpose we have developed a tool based on a web platform that, using open source technology, is able to acquire, manage, aggregate and allow visualization thereof of real time data collected by sensors of MMS. Furthermore a historical track of the data can be logged if necessary.

The web platform is organized on a modular software architecture (Figure 2) so that each sensor of the MMS is managed independently with respect to the others through dedicated modules. This software architecture based on separate blocks offers advantages like:

(i) easy integration of new sensors in the MMS; (ii) usage of different types of open source languages; (iii) integration of existing modules; (iv) team work.

Python programming language was used in the first step of developing the architecture. These collection of modules embody the back-end structure of the web tool and their main roles are summarized in the following keywords: connection, acquisition, interpretation and archiving.

The first step is when the system starts communication with the different sensors using their communication protocol (serial communication protocols).
Once connected, the system is able to program the sensors (if they require) and to set the interval of data transmission.

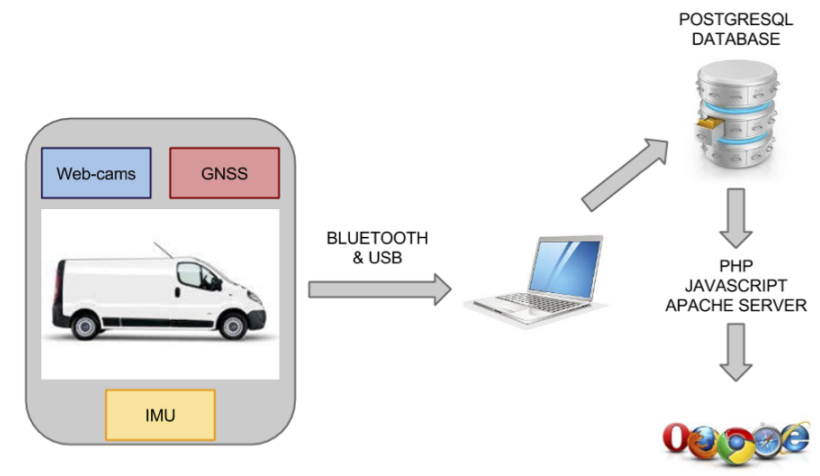

Figure 2. Schematic illustration of system modularity.

Regarding our case, we have scheduled the IMU sensor to send the measures at a frequency of $100 \mathrm{~Hz}$ while for the GPS we have maintained the default sampling rate of the device. About the webcam, we have decided to set the rate of data acquisition at a frame every five seconds in order to reduce the quantity of measure to be stored without losing the information contained in the data.

Once the data transmission mode is set, the system is ready to acquire the output signals of the sensors, and process them for their role in the processing model.

Finally the data collected are archived in a relational database in order to be published in real time on the web tool and to be managed in the post processing phase. We chose a Postgres database organized in multiple tables, in perspective to use its Postgis extension. Each table of the database is structured to have fields to store the output data from sensors with the timestamp at which they were collected.

This part of the software is architected as a command line tool which produced textual summary of measurements to make it possible the supervision of the MMS. This part of the system does not produce by itself a rich graphical output (Figure 2), but it is designed to reduce power consumption in the case of long lasting surveys.

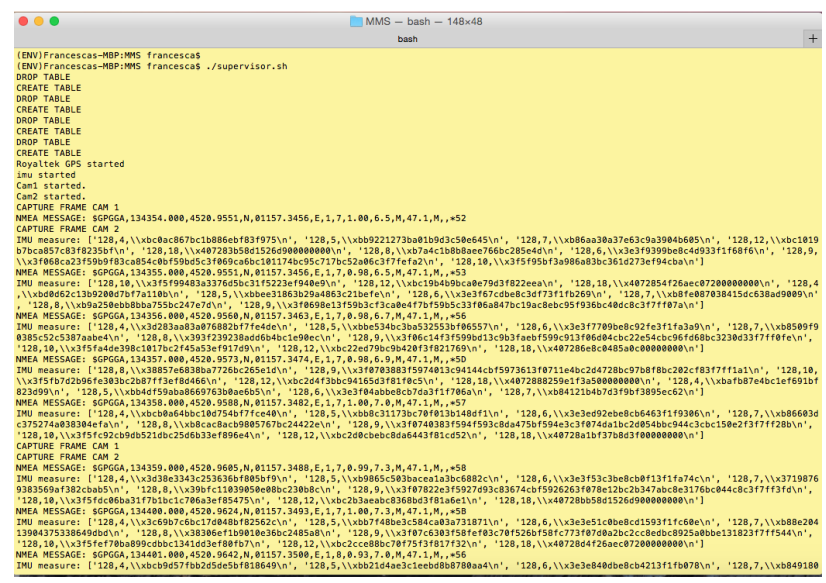

Figure 3. Example of command line tool which produced textual summary of measurements.

In order to facilitate the interaction between the user and the MMS data and to increase the intuitive usage and access, we have 
decided to develop a web interface with clear panels for each part of the MMS information to be accessed by user.

The web interface represents the front end of our project and it is the means by which users manage the data coming from the MMS. Even for the front end we have adopted the concept of open source and modular software architecture with the use of JQuery, Handlebars, Canvas libraries. Through the "Start" button on the homepage, the user initiates the system, and the database is created by functions on the back-end libraries, in line with what discussed in the previous section. Now, surfing the web tool, the user monitors completely and in real time the whole mobile mapping system.

The web platform schema is organized hierarchically with web pages accessible from the initial homepage. The homepage is designed using independent blocks, identified by different colors and showing the main parameters measured by the sensors. Specifically we have reported the values of latitude, longitude and GPS time, the frames captured by the webcam and the main values of navigation e.g. (i) the direction and magnitude of the acceleration, (ii) rate of rotation and (iii) Euler angles.

Limited to the IMU sensor and in addition to real-time data, we have included the sparkline that shows the trends of the navigation parameters.

In order to georeference the position of the MMS, a continuously updated map keeps track of the distance covered during the survey mission of mobile mapping. An example of homepage is visible in Figure 4.

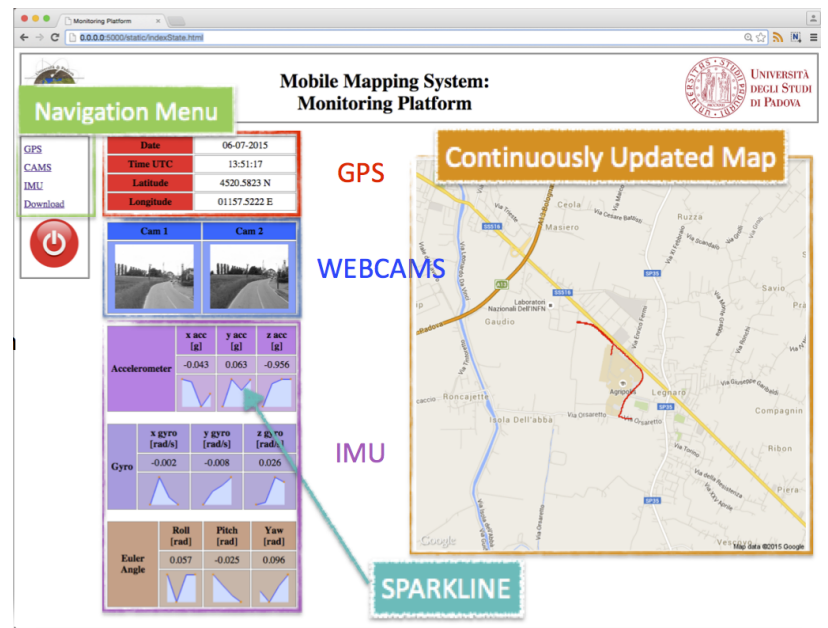

Figure 4. The Homepage of the Mobile Mapping web tool.

Through the navigation menu the users have access to the web pages devoted to the single type of sensors where the values of all parameters acquired by the device are visible in real time. An example is shown in Figure 5.

To better monitor the navigation parameters, we show the trend in time of the IMU sensor parameters (Figure 6). This graph is upgraded every second and it is structured to display in a single plot the three components of the inertial navigation apparatus.

This type of visualization is accessible by clicking the graphs from the homepage or from the dedicated page.

Finally in order to facilitate data recovering and use in post processing as open data, we have created a special section of the web

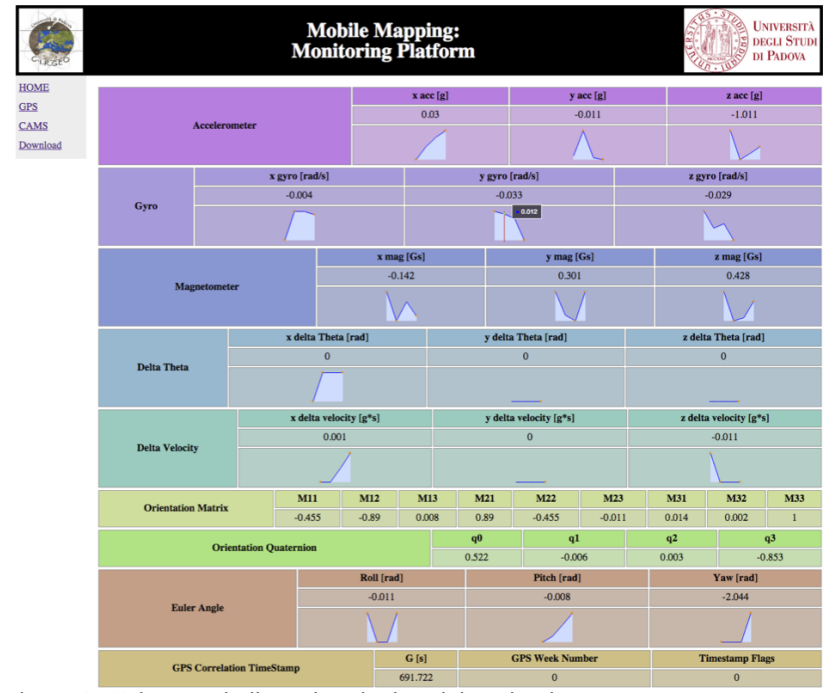

Figure 5. Web page dedicated to the inertial navigation system.

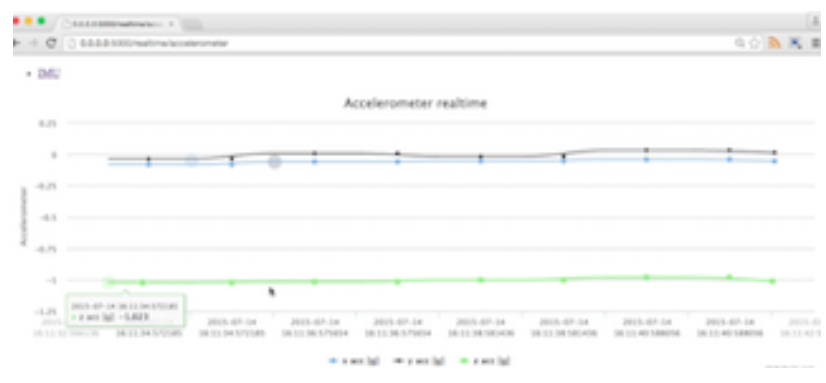

Figure 6. An example of real time measures from the inertial navigation sensor.

tool, accessible from the voice "Download", where the user may get the measures recorded by the single sensors in compressed textfiles.

\section{FUTURE DEVELOPMENTS AND CONCLUSIONS}

This paper presented the project conducted by the CIRGEO group at Padua University to create a low cost Mobile Mapping System and build a platform to manage its data.

The goal of this project is to achieve low cost mobile mapping system leveraging where possible open source libraries for data management. This objective has been reached using low cost sensors and the development of a web tool to manage the mobile mapping system only with open source software.

The use of open source technologies and of a modular architecture have made our system highly flexible and open to the integration of other sensors and to be customized on the base of scientific area of application. In addition the performance of a friendly web tool for its management, has opened potential usage of the data to others, thus initiating what could be seen as a sharing principle and a collaborative potential (Pirotti et al., 2011, Piragnolo et al., 2015).

Possible future developments of our project will focus on the integration of other sensors in our low cost MMS, such as laser scanner and other cameras, in order to differentiate the performance of our low cost system and to allow an accurate reconstruction of the 
environment observed during the survey mission in post processing phase. Moreover we will integrate other modules devoted to the calibration of the sensors in order to validate different methods of calibration (Dong et al., 2007, Heikkila, 2000, Remondino and Fraser, 2006, Syed et al., 2007, Zhang, 1999) and develop new ones. Furthermore, we will focus on the synchronization of the signals from sensors. In the first phase this procedure will be carried out during post processing, but later it will be integrated inside the web tool.

Other future developments are related to exploiting data collected by the presented MMS in order to test the performance of a free photogrammetric tool under development at the University of Padua for both calibrated and uncalibrated cameras (Masiero and Vettore, 2016), i.e. self- calibration (Heikkila, 2000, Remondino and Fraser, 2006, Reznicek, 2014, Zhang, 1999) and their use for monitoring lands and infrastructures, e.g. (Guarnieri et al., 2015, Pirotti et al., 2015, Masiero et al., 2015).

\section{REFERENCES}

Dong, Z., Zhang, G., Luo, Y., Tsang, C. C., Shi, G., Kwok, S. Y., Li, W. J., Leong, P. H. W. and Wong, M. Y., 2007. A calibration method for MEMS inertial sensors based on optical tracking. In: 2007 2nd IEEE International Conference on Nano/Micro Engineered and Molecular Systems, pp. 542-547.

Ellum, C. and El-Sheimy, N., 2002. Land-based mobile mapping systems. Photogrammetric engineering and remote sensing 68(1), pp. 13-17.

Fissore, F., Masiero, A., Piragnolo, M., Pirotti, F., Guarnieri, A. and Vettore, A., 2018. Towards surveying with a smartphone. In: New Advanced GNSS and 3D Spatial Techniques, Springer, pp. 167-176.

Guarnieri, A., Masiero, A., Vettore, A. and Pirotti, F., 2015. Evaluation of the dynamic processes of a landslide with laser scanners and bayesian methods. Geomatics, Natural Hazards and Risk 6(57), pp. 614-634.

Guarnieri, A., Menin, A., Pirotti, F. and Vettore, A., 2008. Low cost system: GPS/MEMS for positioning. In: Proceedings of FIG Working Week, pp. 14-19.

Heikkila, J., 2000. Geometric camera calibration using circular control points. IEEE Transactions on pattern analysis and machine intelligence 22(10), pp. 1066-1077.

Leica Pegasus backpack, $2016 . \quad$ http://leicageosystems.com/products/mobile-sensor-platforms/captureplatforms/leica-pegasus-backpack. Accessed: 2017-10-19.

Li, R., 1997. Mobile mapping: An emerging technology for spatial data acquisition. Photogrammetric Engineering and Remote Sensing 63(9), pp. 1085-1092.

Lo, C., Tsai, M., Chiang, K., Chu, C., Tsai, G., Cheng, C., ElSheimy, N. and Ayman, H., 2015. The direct georeferencing application and performance analysis of UAV helicopter in GCPfree area. The International Archives of Photogrammetry, Remote Sensing and Spatial Information Sciences 40(1), pp. 151.

Masiero, A. and Vettore, A., 2016. Improved feature matching for mobile devices with IMU. Sensors 16(8), pp. 1243.

Masiero, A., Fissore, F. and Vettore, A., 2017. A low cost UWB based solution for direct georeferencing UAV photogrammetry. Remote Sensing 9(5), pp. 414.
Masiero, A., Guarnieri, A., Pirotti, F. and Vettore, A., 2015. Semi-automated detection of surface degradation on bridges based on a level set method. ISPRS - International Archives of Photogrammetry, Remote Sensing and Spatial Information Sciences 40(3), pp. 15-21.

Nex, F. and Remondino, F., 2014. UAV for 3D mapping applications: a review. Applied Geomatics 6(1), pp. 1-15.

Petrie, G., 2010. An introduction to the technology mobile mapping systems. Geoinformatics 13(1), pp. 32-43.

Piragnolo, M., Masiero, A., Fissore, F. and Pirotti, F., 2015. Solar irradiance modelling with NASA WW GIS environment. ISPRS International Journal of Geo-Information 4(2), pp. 711-724.

Piras, M., Cina, A. and Lingua, A., 2008. Low cost mobile mapping systems: an italian experience. In: Position, Location and Navigation Symposium, 2008 IEEE/ION, IEEE, pp. 1033-1045.

Pirotti, F., Guarnieri, A. and Vettore, A., 2011. Collaborative web-GIS design: A case study for road risk analysis and monitoring. Transactions in GIS 15(2), pp. 213-226.

Pirotti, F., Guarnieri, A., Masiero, A. and Vettore, A., 2015. Preface to the special issue: the role of geomatics in hydrogeological risk. Geomatics, Natural Hazards and Risk 6(5-7), pp. 357-361.

Puente, I., González-Jorge, H., Martínez-Sánchez, J. and Arias, P., 2013. Review of mobile mapping and surveying technologies. Measurement 46(7), pp. 2127-2145.

Remondino, F. and Fraser, C., 2006. Digital camera calibration methods: considerations and comparisons. International Archives of Photogrammetry, Remote Sensing and Spatial Information Sciences 36(5), pp. 266-272.

Reznicek, J., 2014. Method for measuring lens distortion by using pinhole lens. The International Archives of Photogrammetry, Remote Sensing and Spatial Information Sciences 40(5), pp. 509.

Schwarz, K. P. and El-Sheimy, N., 2004. Mobile mapping systems-state of the art and future trends. International Archives of Photogrammetry, Remote Sensing and Spatial Information Sciences 35(Part B), pp. 10.

Syed, Z., Aggarwal, P., Goodall, C., Niu, X. and El-Sheimy, N., 2007. A new multi-position calibration method for MEMS inertial navigation systems. Measurement Science and Technology 18(7), pp. 1897

Tao, C. V., 2000. Mobile mapping technology for road network data acquisition. Journal of Geospatial Engineering 2(2), pp. 114

Tao, C. V. and Li, J., 2007. Advances in mobile mapping technology. Vol. 4, CRC Press.

Zhang, Z., 1999. Flexible camera calibration by viewing a plane from unknown orientations. In: Computer Vision, 1999. The Proceedings of the Seventh IEEE International Conference on, Vol. 1, Ieee, pp. 666-673.

\section{APPENDIX}

\subsection{Precision and Accuracy of low cost GPS sensor}

In order to test the performance of the low cost GPS included into our MMS, we conducted a field test on horizontal and vertical position errors of our sensor. 
The test was executed placing the device in a known point of the courtyard of university campus of Agripolis in Legnaro (PD Italy) at latitude of $45^{\circ} 20^{\prime} 48.1^{\prime \prime} \mathrm{N}$ and longitude of $11^{\circ} 57^{\prime} 26.9^{\prime \prime} \mathrm{E}$ with an elevation of $52 \mathrm{~m}$ above sea level and away from obstacles such as trees of buildings.

Before starting the test, the reference coordinate of positioning point was determined as an average of fixed resolutions, obtained by static surveying with dual-frequency GPS receiver (TOPCOM) Moreover, to have a better resolution of the positioning point, the coordinates collected in static surveying was corrected in real time, using the nearest permanent GNSS station site at $45^{\circ} 11^{\prime} 6^{\prime}$ 'N $11^{\circ} 16^{\prime} 30^{\prime \prime} \mathrm{E}$ and at $71 \mathrm{~m}$ above the sea level.

The test was performed in April, in two consecutive days, in order to reduce the GPS positional error due to the variation ionosphere and atmosphere conditions. The GPS position was repeated for 6 times, with an observation period of 20 minutes for each section (Table1). In addition the 6 observations of the positioning point was repeated at different times of the days to remove the GPS errors from the geometric distribution of GPS satellites. Finally the test was done only when the number of satellites was equal to or more then 5 .

\begin{tabular}{|c|c|c|}
\hline Date & Time & Starting Time \\
\hline \hline \multirow{3}{*}{$14 / 04 / 2016$} & 1 & $9: 10: 52$ \\
& 2 & $12: 03: 10$ \\
& 3 & $16: 15: 32$ \\
\hline \multirow{3}{*}{$15 / 04 / 2016$} & 4 & $9: 16: 37$ \\
& 5 & $12: 06: 58$ \\
& 6 & $16: 04: 21$ \\
\hline
\end{tabular}

Table 1. Times of observation period.

\subsection{Definitions of positioning errors}

In this study we have used the root mean square (RMS) to estimate the GPS positional errors in term of precision and accuracy.

With the term accuracy we refer to the closeness of the sample mean to the true value, while with precision we relate to the closeness of repeated observation to sample mean.

4.2.1 Horizontal positional errors For RMS calculations, horizontal precision was calculated by (1)

$$
\sigma_{H_{\text {precision }}}=\sqrt{\sigma_{N}^{2}+\sigma_{E}^{2}}
$$

where $\sigma_{H_{\text {precision }}}$ indicates RMS; while $\sigma_{N}^{2}$ and $\sigma_{E}^{2}$ indicate the standard deviation of positional error along Northing and Easting directions respectively, and are calculated by (2) and (3).

$$
\begin{aligned}
\sigma_{N}^{2} & =\frac{\sum_{i=1}^{n}\left(N_{i}-\bar{N}\right)^{2}}{n-1} \\
\sigma_{E}^{2} & =\frac{\sum_{i=1}^{n}\left(E_{i}-\bar{E}\right)^{2}}{n-1}
\end{aligned}
$$

Where $n$ is the total number of epochs, $E_{i}$ and $N_{i}$ indicate the location of ith epoch along Northing and Easting direction respectively; $\bar{E}$ and $\bar{N}$ are the sample mean of the measurements along Northing and Easting direction.

On the other hand, horizontal accuracy was calculated using the following equation:

$$
\sigma_{H_{\text {accuracy }}}=\sqrt{\left(\bar{N}-N_{\text {true }}\right)^{2}+\left(\bar{E}-E_{\text {true }}\right)^{2}}
$$

4.2.2 Vertical positional errors Vertical positional errors were calculated by the following equations:

$$
\begin{gathered}
\sigma_{V_{\text {precision }}}=\sqrt{\frac{\sum_{i=1}^{n}\left(V_{i}-\bar{V}\right)^{2}}{n-1}} \\
\sigma_{V_{\text {accuracy }}}=\left|\bar{V}-V_{\text {true }}\right|
\end{gathered}
$$

Where $\sigma_{V_{\text {precision }}}, \sigma_{V_{\text {accuracy }}}$ indicate vertical precision and accuracy; $\bar{V}$ is the sample mean of the positional error along vertical axis; $V_{\text {true }}$ is the true location along the vertical axis.

\section{RESULTS AND CONCLUSION}

We have collected 20 minutes of GPS data in the 6 different times. For each of these samples we have estimated the horizontal and vertical precision by $(1,4,5,6)$.

The horizontal precision and accuracy results are shown in Table 2.

\begin{tabular}{|c|c|c|}
\hline Time & Precision $(\mathrm{m})$ & Accuracy $(\mathrm{m})$ \\
\hline \hline 1 & 5.33 & 1.4 \\
2 & 5.45 & 1.76 \\
3 & 4.42 & 1.25 \\
4 & 3.88 & 6.63 \\
5 & 2.35 & 7.69 \\
6 & 8.03 & 3.25 \\
\hline
\end{tabular}

Table 2. Horizontal positional errors.

As shown in the Table 2, the smallest and largest precision errors were recorded in the second day at the 5 and 6 time, respectively. Regarded to accuracy error was smallest in the afternoon of the first day, while the largest was in the second day at the 5 time, same time in whish was recorded the smallest precision error.

Vertical precision and accuracy errors at the 6 times are shown in Table 3

\begin{tabular}{|c|c|c|}
\hline Time & Precision $(\mathrm{m})$ & Accuracy $(\mathrm{m})$ \\
\hline \hline 1 & 0.00 & 3.31 \\
2 & 0.00 & 3.31 \\
3 & 0.00 & 3.31 \\
4 & 0.00 & 3.31 \\
5 & 0.00 & 3.37 \\
6 & 0.00 & 3.37 \\
\hline
\end{tabular}

Table 3. Vertical positional errors .

As shown in Table 3 the precision errors of our GPS were the same for all the times and they were very smallest.Regarding the accuracy errors, there were the same for the times of the same day.

The main effects of the GPS positioning points on horizontal and vertical precision and accuracy are shown in Figures $(7,8)$ and summarized in Table 4.

In conclusion, the low cost GPS using in the project have an accuracy of $4(\mathrm{~m})$ and $3(\mathrm{~m})$ along horizontal and vertical position. Instead The precision of the sensor is a $5(\mathrm{~m})$ in horizontal direction while negligible in the other dimension. 
The International Archives of the Photogrammetry, Remote Sensing and Spatial Information Sciences, Volume XLII-2/W8, 2017 5th International Workshop LowCost 3D - Sensors, Algorithms, Applications, 28-29 November 2017, Hamburg, Germany

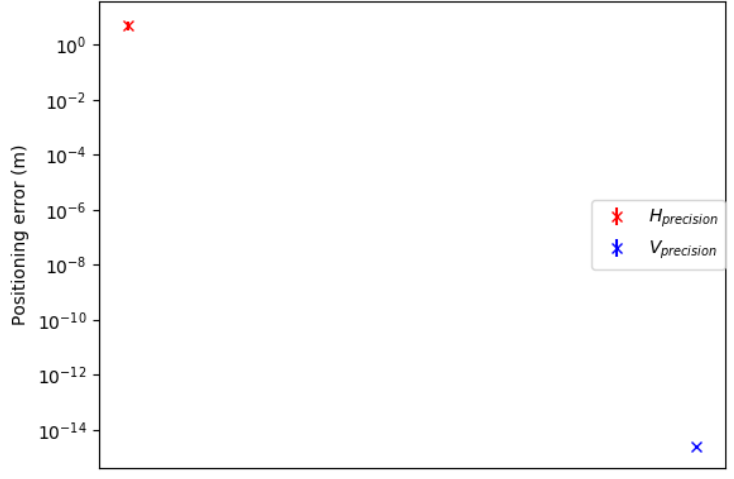

Figure 7. Precision of positioning error.

\begin{tabular}{|c|c|}
\hline$H_{\text {precision }}$ & $4.91 \pm 1.73(\mathrm{~m})$ \\
$H_{\text {accuracy }}$ & $3.66 \pm 2.57(\mathrm{~m})$ \\
\hline$V_{\text {precision }}$ & $2.37 e^{-15} \pm 3.35 e^{-15}(\mathrm{~m})$ \\
$V_{\text {accuracy }}$ & $3.33 \pm 0.023(\mathrm{~m})$ \\
\hline
\end{tabular}

Table 4. Values of precision and accuracy error.

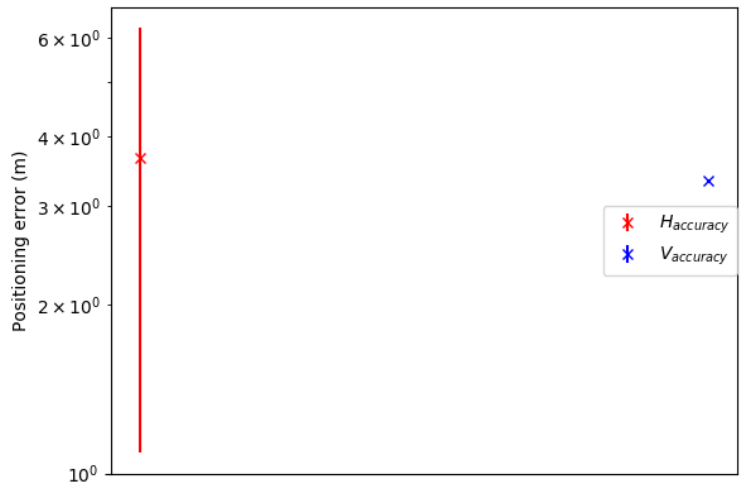

Figure 8. Accuracy of positioning error. 